\title{
Leiomiosarcoma dérmico y subcutáneo: actualización y controversias, a propósito de un caso
}

\section{Dermal and subcutaneous leiomyosarcoma: update and controversies, due to a case}

\author{
Francisco J. Melgosa-Ramos ${ }^{1 *}$, Andrea Estébanez-Corrales ${ }^{1}$, Enrique Salmerón-González², \\ Encarna Martí-Ibor ${ }^{3}$, Eva M. Sánchez-Martínez ${ }^{1}$ y Almudena Mateu-Puchades ${ }^{1}$ \\ ${ }^{1}$ Servicio de Dermatología; ${ }^{2}$ Servicio de Cirugía Plástica, estética y reparadora; ${ }^{3}$ Servicio de Anatomía Patológica. Hospital Universitario Doctor \\ Peset. Valencia, España
}

\section{Resumen}

El leiomiosarcoma cutáneo (LMSC) es una neoplasia maligna poco común que supone menos del $0.05 \%$ del total de los tumores cutáneos, mucho menos frecuente que otros sarcomas como el de Kaposi o el dermatofibrosarcoma protuberans. Se distinguen tres subtipos con diferente manejo y pronóstico: el leiomiosarcoma dérmico, el leiomiosarcoma subcutáneo y el leiomiosarcoma metastásico. Esta clasificación, a priori sencilla, no siempre permite encuadrar el LMSC como dérmico o subcutáneo, ocasionando cierta heterogeneidad en algunos aspectos, como el estudio de extensión, el manejo quirúrgico y la necesidad o no de terapia adyuvante. La baja frecuencia de estos tumores no ayuda a lograr un manejo estandarizado en la práctica diaria. Presentamos un caso de LMSC que permite discutir los interrogantes planteados.

Palabras clave: Leiomiosarcoma cutáneo. Leiomiosarcoma dérmico. Leiomiosarcoma tratamiento.

\begin{abstract}
Cutaneous leiomyosarcoma is an uncommon malignant neoplasm that represents less than $0.05 \%$ of skin tumors, much less frequent than other sarcomas such as Kaposi sarcoma or dermatofibrosarcoma protuberans. Three main subtypes are distinguished with different management and prognosis: dermal leiomyosarcoma, subcutaneous leiomyosarcoma, and metastatic leiomyosarcoma. Despite its simplicity, this classification does not always allow to classify a LMSC as dermal or subcutaneous, causing certain variability in some aspects such as the extension study, surgical management, and the adjuvant therapy. In addition, the low prevalence of these tumors does not help to achieve standardized management on daily clinical practice. We present a case of LMSC that allows us to discuss the questions raised.
\end{abstract}

Keywords: Cutaneous Leiomyosarcoma. Dermal Leiomyosarcoma. Leiomyosarcoma treatment.

\section{Introducción}

El leiomiosarcoma cutáneo (LMSC) es una neoplasia infrecuente resultante de la proliferación de células malignas de estirpe muscular. Representa menos del
$0.05 \%$ del total de las neoplasias cutáneas y en torno al 2-3\% de los sarcomas cutáneos ${ }^{1}$, estando en frecuencia por detrás de otros como el sarcoma de Kaposi y el dermatofibrosarcoma protuberans ${ }^{1}$. Clásicamente se distinguen tres tipos principales: leiomiosarcoma
Correspondencia:

${ }^{\star}$ Francisco J. Melgosa-Ramos

E- mail: javimelgo2017@gmail.com

DOI: 10.24875/MCUT.21000033
Disponible en internet: 28-01-2022 Med Cutan Iber Lat Am. 2022;50(1):23-28 www.MedicinaCutanealLA.com licencia CC BY-NC-ND (http://creativecommons.org/licenses/by-nc-nd/4.0/). 
dérmico, leiomiosarcoma subcutáneo y leiomiosarcoma metastásico; todos ellos con diferentes manejo y pronóstico. Aunque esta clasificación parece sencilla, no siempre es fácil clasificar un LMSC como dérmico o subcutáneo, lo cual genera controversia sobre los estudios de extensión necesarios, el manejo quirúrgico y la necesidad o no de tratamiento adyuvante. La baja frecuencia de estos tumores tampoco ayuda a conseguir un manejo estandarizado. A continuación, presentamos un caso de LMSC que permite discutir los interrogantes planteados.

\section{Caso clínico}

Varón de 51 años remitido a consultas de dermatología por una lesión en la cara lateral de la pierna derecha, de crecimiento progresivo, de varios años de evolución. El paciente refería una intervención quirúrgica en la zona por una fractura de tibia 15 años atrás, con aparición de la lesión sobre la zona cicatricial. En la exploración física se observaba una lesión nodular de color amarillo anaranjado, de $5 \mathrm{~cm}$ de diámetro, en la cara lateral de la pierna derecha, con la base infiltrada y algo dolorosa al tacto (Fig. 1). Con la sospecha clínica de sarcoma de partes blandas o carcinoma epidermoide, se decidió tomar biopsias en zonas diferentes de la lesión. Histológicamente se apreciaba una tumoración positiva para actina y desmina, pero sin atipia ni mitosis, sin poder concluir el carácter benigno o maligno de la lesión. Dada la sospecha clínica de malignidad, 3 semanas después se realizó una nueva biopsia, más amplia y profunda, y se realizaron una resonancia magnética (RM) local de la zona y una radiografía de tórax. En la nueva biopsia se observó una proliferación de células fusocelulares en la dermis superficial y profunda, dispuestas en fascículos arremolinados, con inmunohistoquímica negativa para CD34, S100, citoqueratinas de amplio espectro A1/A3, y positiva para actina y desmina, todo ello compatible con leiomiosarcoma, a determinar tras la exéresis completa el carácter dérmico o subcutáneo. La radiografía de tórax fue normal y la RM mostró extensión al tejido subcutáneo y probable invasión de la fascia muscular del peroneo corto. Ante la posibilidad de un LMSC subcutáneo, se solicitó una tomografía computarizada (TC) toracoabdominopélvica que descartó enfermedad a distancia. Tras discusión en comité multidisciplinario, se decidió realizar una exéresis amplia con márgenes de $3 \mathrm{~cm}$ y resección completa de peroneos cortos y largos (Figs. 2 y 3). El defecto se reconstruyó con un colgajo libre

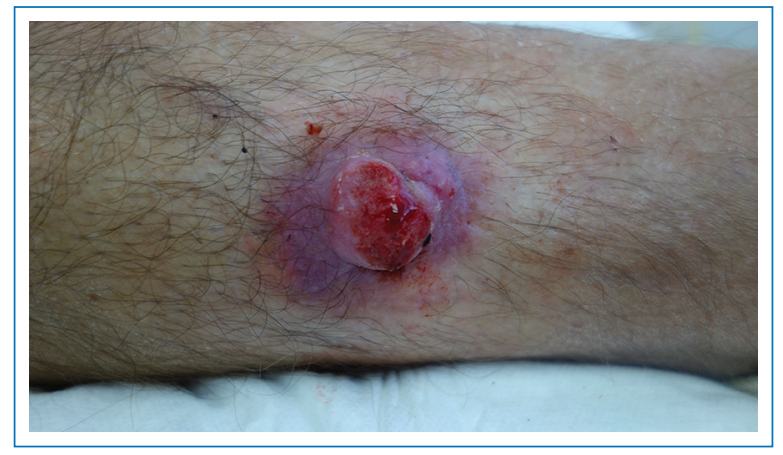

Figura 1. Lesión nodular de color amarillo anaranjado, de $5 \mathrm{~cm}$ de diámetro, en la cara lateral de la pierna derecha.

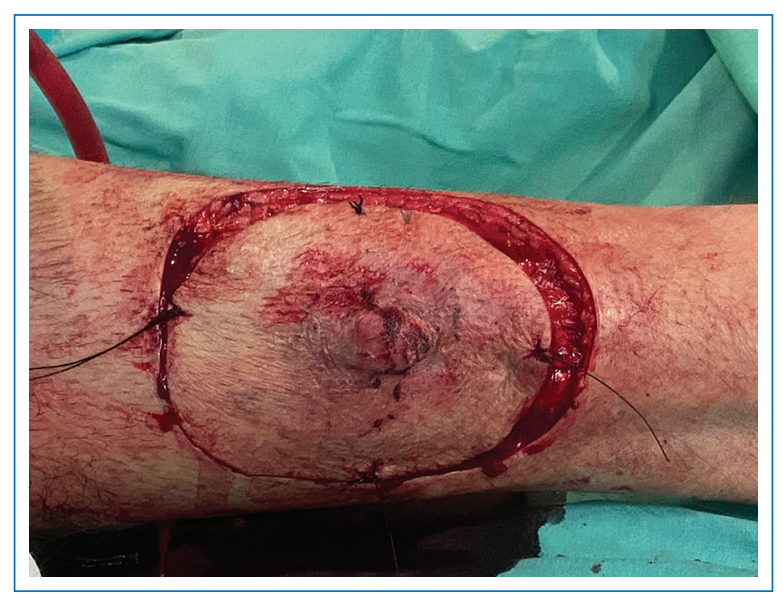

Figura 2. Resección quirúrgica con márgenes.

microvascularizado de vasto lateral con injerto de piel parcial, con buena evolución posoperatoria (Fig. 4). El examen histopatológico definitivo confirmó el diagnóstico de LMSC dérmico con extensión al tejido celular subcutáneo, con cuatro mitosis por campo de gran aumento, bordes de resección libres, sin invasión linfovascular ni perineural, y ausencia de necrosis y de áreas mixoides. La inmunohistoquímica fue positiva para actina, desmina y h-caldesmón (Figs. 5 a 7). Dada la invasión en profundidad, el tamaño de la lesión $(>5 \mathrm{~cm}$ ) y la localización acral, se planteó la posibilidad de radioterapia adyuvante; sin embargo, tras la discusión en comité multidisciplinario se decidió realizar seguimiento clínico estrecho sin radioterapia adyuvante, pues como a continuación exponemos, el beneficio no está del todo claro y, sin embargo, complicaría una reintervención en caso de recidiva. 


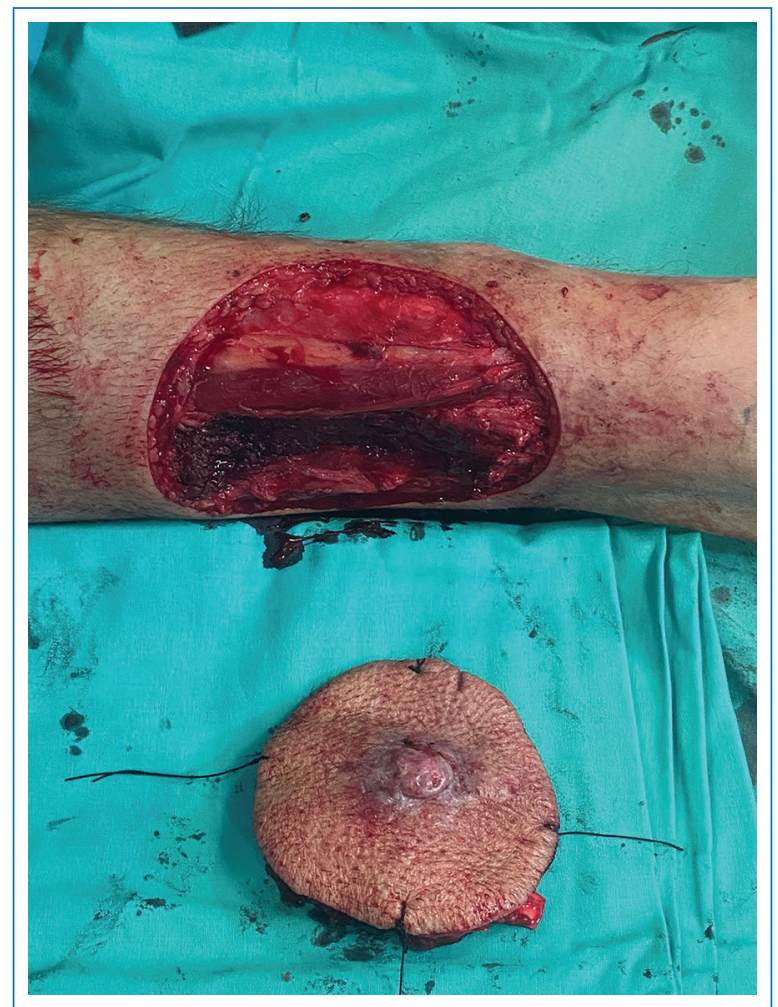

Figura 3. Pieza quirúrgica extirpada, con exposición de la musculatura local.

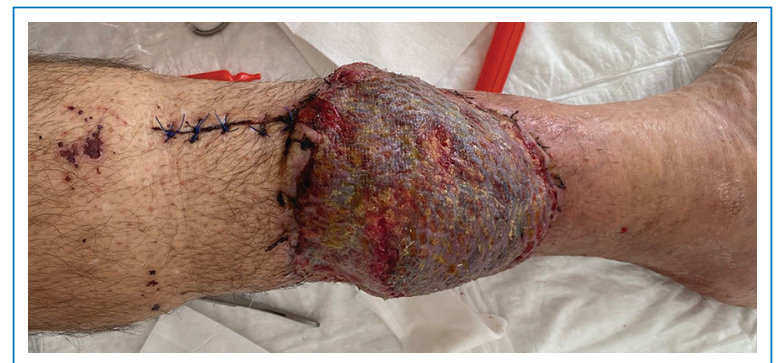

Figura 4. Colgajo libre microvascularizado de vasto lateral con injerto de piel parcial (primeros días de posoperatorio).

\section{Discusión}

El LMSC supone en torno al 2-3\% de los sarcomas cutáneos y menos del $0.05 \%$ del total de las neoplasias cutáneas ${ }^{1}$. El LMSC dérmico se origina del músculo erector del pelo, el dartos genital o areolar, mientras que el subcutáneo se origina de la capa muscular de los vasos del tejido celular subcutáneo y los casos metastásicos cutáneos suelen tener un origen visceral, con predominio retroperitoneal ${ }^{1,2}$. El LMSC dérmico se define como aquella tumoración predominantemente

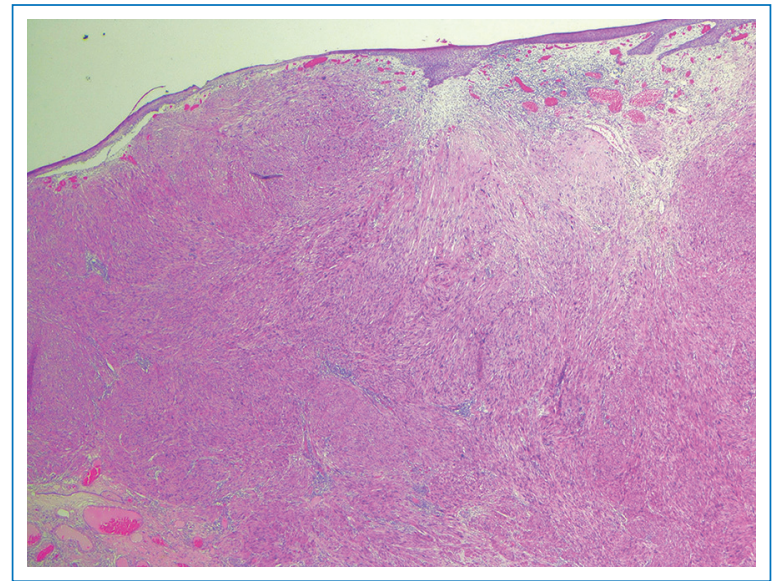

Figura 5. Imagen histopatológica (hematoxilina-eosina 2x) que muestra proliferación tumoral ocupando la dermis superficial y profunda.

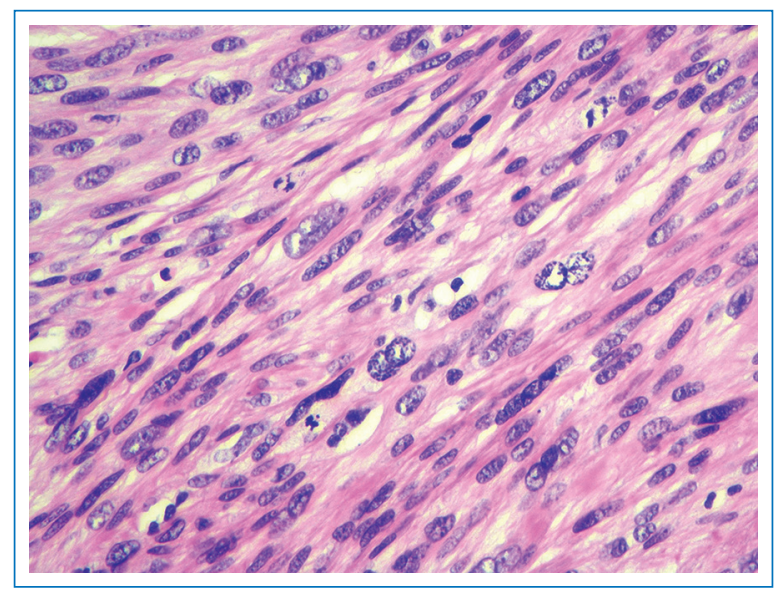

Figura 6. Proliferación de células fusiformes con atipia citológica.

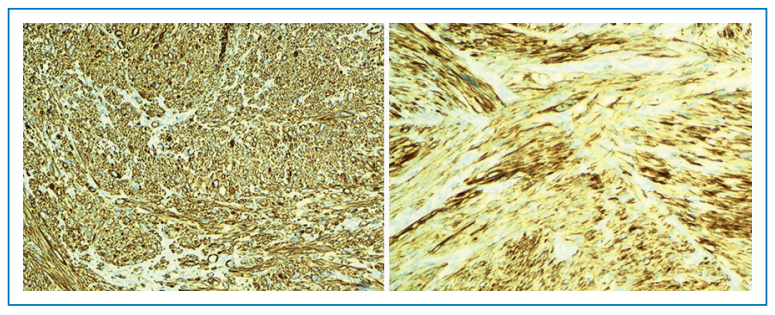

Figura 7. Tinción positiva para h-caldesmón (izquierda) y desmina (derecha).

dérmica con o sin invasión limitada del tejido celular subcutáneo. Algunos autores subdividen este grupo en tumor atípico intradérmico de músculo liso, con 
prácticamente nula capacidad de extensión a distancia, y leiomiosarcoma "dérmico» con posible extensión al tejido celular subcutáneo y mínima capacidad metastásica ${ }^{2,3}$. Los tumores predominantemente subcutáneos, que pueden llegar a comprometer la fascia 0 extenderse de forma limitada a la dermis, son considerados leiomiosarcomas subcutáneos. El pronóstico y la capacidad metastásica dependen de la profundidad (en torno al $4 \%$ de los LMSC dérmicos frente al $43 \%$ de los subcutáneos producen metástasis) $)^{1,4}$, siendo el pulmón, la piel y el territorio ganglionar las localizaciones de metástasis más frecuentes.

La edad de presentación es variable; la mayoría de los trabajos publicados coinciden en el predominio en la franja de 50 a 70 años, y afectan con más frecuencia a los varones (3:1) de raza blanca ${ }^{1,2,5}$. La presentación clínica es algo inespecífica y común en ambos subtipos. Lo más habitual es la aparición de una lesión nodular única, de crecimiento lento, consistencia firme y color variable (rosado, marronáceo o como la piel circundante); sin embargo, también pueden presentarse como placas con o sin nódulos en su superficie, o como lesiones que simulan lipomas. Las lesiones pueden ser asintomáticas, pero es muy característico que duelan a la palpación, siendo más raro el dolor espontáneo. Habitualmente, el LMSC subcutáneo está mejor delimitado que el dérmico. La localización más frecuente es en la superficie de extensión de las extremidades (> 50-60\%), sobre todo en el caso de las lesiones subcutáneas. Entre los potenciales desencadenantes se han planteado varios, como antecedentes de radioterapia local, traumatismos e incluso una intervención quirúrgica previa en la zona, sin evidencia suficiente al respecto $0^{1,2,5,6}$.

El diagnóstico de certeza, dada la inespecificidad clínica, es histopatológico, por lo que es necesario tomar muestras profundas que incluyan tejido celular subcutáneo. Pueden ser necesarias varias biopsias para alcanzar el diagnóstico. El LMSC dérmico es una lesión mal delimitada constituida por células musculares lisas fusiformes de citoplasma eosinófilo con ocasional extensión al tejido celular subcutáneo'. EI LMSC subcutáneo es una lesión mejor definida, en principio limitada a la grasa, pero que con el tiempo puede producir invasión profunda e incluso ocupación dérmica, generando ciertas dudas en casos avanzados como el nuestro. Algunos autores defienden la existencia de dos patrones de crecimiento, uno nodular, con más celularidad, atipia y mitosis, y otro difuso, que puede llegar a confundirse con lesiones benignas ${ }^{1,7,8}$. En algunos casos, como el que presentamos, la toma de una o varias biopsias, aun siendo profundas, no permite clasificar la lesión como dérmica o subcutánea, y es solo tras la exéresis completa cuando se puede apreciar si la tumoración es predominantemente dérmica o subcutánea. Esto puede implicar cierta incertidumbre en cuanto al estudio de extensión o el planteamiento quirúrgico.

La inmunohistoquímica también puede desempeñar un papel clave en el diagnóstico, sobre todo para diferenciar el LMSC de otros sarcomas y tumores de células fusiformes. El LMSC normalmente es positivo para h-caldesmón, vimentina, actina y miosina, y variable para desmina, la cual puede ser negativa en LMSC subcutáneos y dérmicos muy indiferenciados, pese a que en algunas series, como la de Wong et al. ${ }^{2}$, no se han encontrado diferencias. Para el diagnóstico es necesaria la positividad de al menos dos de estos marcadores. La negatividad de otros marcadores propios de tumores de células fusiformes, como CD34, HMB45 y CK7, entre otros, apoya el diagnóstico, mientras que el S100 muestra positividad variable $e^{1,2,9,10}$.

La estadificación también es compleja, ya que no existe un sistema TNM propio. A ello se suman las diferentes opiniones respecto a las pruebas complementarias necesarias, sobre todo en el caso del LMSC dérmico. El grupo de Llombart et al. ${ }^{1}$ destaca la utilidad de la RM preoperatoria, el posible empleo de la ecografía y la realización de radiografía de tórax prequirúrgica. Wong et $\mathrm{al}^{2}$ realizan estudio de extensión en el $58 \%$ de los LMSC dérmicos, mientras que Deneve et al. ${ }^{11}$ comentan el poco impacto de algunas de estas pruebas en el caso del LMSC dérmico, pues en los 33 casos de su serie el estudio de extensión fue negativo. A pesar de las diferentes posiciones, parece prudente solicitar un estudio de extensión teniendo en cuenta el potencial metastásico de estos tumores. En cuanto al LMSC subcutáneo, las recomendaciones parecen más uniformes debido a su mayor potencial invasor y metastásico, recomendando la realización de RM preoperatoria y el estudio de imagen con TC de tórax y abdomen superior (en algunas series se ha utilizado tomografía por emisión de positrones), lo que permite además cribar el origen en un leiomiosarcoma oculto ${ }^{1,3,12}$.

En cuanto al tratamiento y el pronóstico, parece haber uniformidad en la parte quirúrgica, pasando de exéresis con márgenes muy amplios, recomendadas hace años, a resecciones con márgenes de $1-3 \mathrm{~cm}$ sin aumentar las recidivas locales. En los LMSC dérmicos superficiales se considera la cirugía de Mohs. El margen profundo debe llegar a la fascia y, si es necesario, incluir músculo y otras estructuras ${ }^{1,3,13}$. 


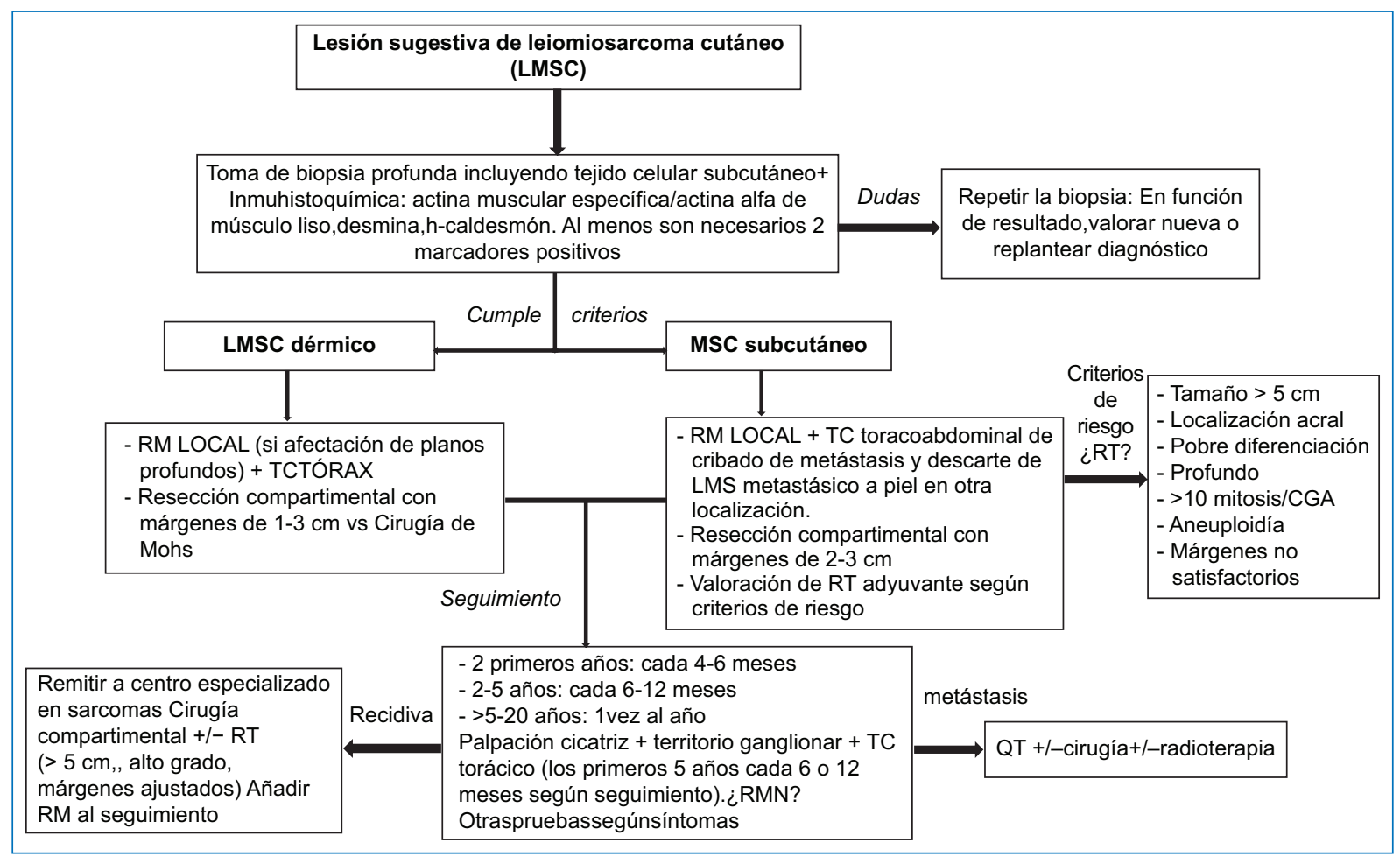

Figura 8. Algoritmo de manejo (adaptado del propuesto por Llombart, et al ${ }^{1}$.). CGA: campo de gran aumento; RM: resonancia magnética; RT: radioterapia; TC: tomografía computarizada.

Si bien las recomendaciones respecto al tratamiento quirúrgico están bastante claras, no existe tanta uniformidad para decidir o no la necesidad de tratamiento adyuvante con radioterapia, pues algunas series concluyen que no disminuye de manera significativa el riesgo de recidiva local. Otros autores defienden, sin poder alcanzar un consenso, que su uso podría estar justificado en recidivas y en caso de LMSC subcutáneos profundos, o con factores de mal pronóstico asociados (tamaño $>5 \mathrm{~cm}$, localización acral, alto número de mitosis definidas como $>10$ por campo de gran aumento, escasa diferenciación, márgenes insuficientes 0 aneuploidía celular, entre otros) ${ }^{1,3,8}$. Resultaría interesante la elaboración de escalas que ayudasen a la toma de decisiones en este grupo. Mientras tanto, parece haber poca justificación para el empleo de radioterapia adyuvante en los LMSC dérmicos superficiales resecados con márgenes adecuados ${ }^{3}$, aunque sí resultaría útil para el control paliativo de las metástasis, en el cual también la quimioterapia, sobre todo con antraciclinas, taxanos o ciclofosfamida, puede ayudar a enlentecer la progresión ${ }^{1,14}$.

En relación con el seguimiento, tampoco existen recomendaciones claras. La tasa de recidiva local de los LMSC dérmicos oscila entre el $18 \%$ y el $24 \%$, según las series, y llega hasta el $37 \%$ en los LMSC subcutáneos $^{1,3,15}$, siendo más frecuentes en los primeros 3 años, aunque se han descrito recaídas tardías después de 10-15 años. Por tanto, resulta necesario realizar un seguimiento más estrecho al menos durante los primeros 5 años. En general, las revisiones se realizan con una periodicidad de 4-6 meses los primeros 2 años, incluyendo estudio con prueba de imagen, como TC de tórax (algunos autores contemplan en sus trabajos la radiografía), y luego con una periodicidad anual los siguientes 3 años (también con TC). Posteriormente, debido a la posibilidad de recaídas tardías, puede ser conveniente continuar el seguimiento clínico hasta los 20 años, sin necesidad de pruebas sistemáticas, solo solicitándolas según los hallazgos exploratorios ${ }^{1,3,5,16}$. Algunos trabajos también defienden el posible papel de la RM local en el seguimiento, que sobre todo es útil para aquellos casos recurrentes en los que la cirugía haya sido compleja'.

En conclusión, el LMSC es un tumor infrecuente y en cuyo manejo existen ciertos aspectos conflictivos que pueden dificultar la toma de decisiones, por lo que es deber de la comunidad médica la búsqueda de un consenso que permita unificar criterios que puedan contribuir a la mejoría pronóstica de estos pacientes. En la figura 8 se muestra un algoritmo, modificación 
del propuesto por Llombart et $\mathrm{al}^{1}$, con el objetivo de incluir algunos de estos aspectos comentados (Fig. 8).

\section{Financiamiento}

Los autores declaran no haber recibido financiamiento para este estudio.

\section{Conflicto de intereses}

Los autores declaran no tener conflictos de intereses.

\section{Responsabilidades éticas}

Protección de personas y animales. Los autores declaran que para esta investigación no se han realizado experimentos en seres humanos ni en animales.

Confidencialidad de los datos. Los autores declaran que han seguido los protocolos de su centro de trabajo sobre la publicación de datos de pacientes.

Derecho a la privacidad y consentimiento informado. Los autores declaran que en este artículo no aparecen datos de pacientes.

\section{Bibliografía}

1. Llombart B, Serra-Guillén C, Requena C, Alsina M, Morgado-Carrasco D, Machado I, et al. Leiomyosarcoma and pleomorphic dermal sarcoma: guidelines for diagnosis and treatment. Actas Dermosifiliogr. 2019;110:4-11.
2. Wong GN, Webb A, Gyorki D, McCormack C, Tran P, Ngan SY, et al Cutaneous leiomyosarcoma: dermal and subcutaneous. Australas J Dermatol. 2020;61:243-9.

3. Kraft S, Fletcher CD. Atypical intradermal smooth muscle neoplasms: clinicopathologic analysis of 84 cases and a reappraisal of cutaneous «leiomyosarcoma». Am J Surg Pathol. 2011;35:599-607.

4. Aneiros-Fernández J, Antonio Retamero J, Husein-Elahmed $\mathrm{H}$, Ovalle $\mathrm{F}$, Aneiros-Cachaza J. Primary cutaneous and subcutaneous leiomyosarcomas: evolution and prognostic factors. Eur J Dermatol. 2016;26:9-12.

5. Winchester DS, Hocker TL, Brewer JD, Baum CL, Hochwalt PC, Arpey CJ, et al. Leiomyosarcoma of the skin: clinical, histopathologic, and prognostic factors that influence outcomes. J Am Acad Dermatol. 2014;71:919-25

6. Fields JP, Helwig EB. Leiomyosarcoma of the skin and subcutaneous tissue. Cancer. 1981;47:156-69.

7. Kaddu S, Beham A, Cerroni L, Humer-Fuchs U, Salmhofer W, Kerl H, et al. Cutaneous leiomyosarcoma. Am J Surg Pathol. 1997;21:979-87.

8. Jensen ML, Jensen OM, Michalski W, Nielsen OS, Keller J. Intradermal and subcutaneous leiomyosarcoma: a clinicopathological and immunohistochemical study of 41 cases. J Cutan Pathol. 1996;23:458-63.

9. Oliver GF, Reiman HM, Gonchoroff NJ, Muller SA, Umbert IJ. Cutaneous and subcutaneous leiomyosarcoma: a clinicopathological review of 14 cases with reference to antidesmin staining and nuclear DNA patterns studied by flow cytometry. Br J Dermatol. 1991;124:252-7.

10. Watanabe K, Kusakabe T, Hoshi N, Saito A, Suzuki T. h-Caldesmon in leiomyosarcoma and tumors with smooth muscle cell-like differentiation: its specific expression in the smooth muscle cell tumor. Hum Pathol. 1999;30:392-6.

11. Deneve JL, Messina JL, Bui MM, Marzban SS, Letson GD, Cheong D, et al. Cutaneous leiomyosarcoma: treatment and outcomes with a standardized margin of resection. Cancer Control. 2013;20:307-12.

12. Llombart B. Cutaneous leiomyosarcoma: on the importance of histologic location. Actas Dermosifiliogr. 2018:109:98.

13. Starling $\mathrm{J} 3^{\text {rd }}$, Coldiron BM. Mohs micrographic surgery for the treatment of cutaneous leiomyosarcoma. J Am Acad Dermatol. 2011;64:1119-22.

14. García del Muro X, de Álava E, Artigas V, Bague S, Brana A, Cubedo R, et al. Clinical practice guidelines for the diagnosis and treatment of patients with soft tissue sarcoma by the Spanish group for research in sarcomas (GEIS). Cancer Chemother Pharmacol. 2016;77: 133-46.

15. Winchester DS, Hocker TL, Roenigk RK. Skin metastases of leiomyosarcoma (LMS): a retrospective review of 21 cases. J Am Acad Dermatol. 2015;72:910-92

16. Massi D, Franchi A, Alos L, Cook M, Di Palma S, Enguita AB, et al. Primary cutaneous leiomyosarcoma: clinicopathological analysis of 36 cases. Histopathology. 2010;56:251-62. 